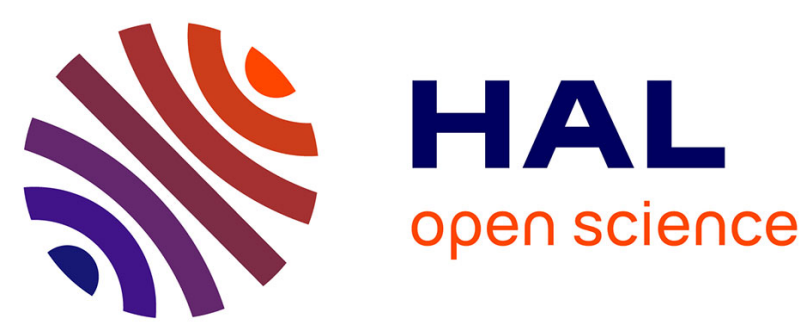

\title{
Inadequate reporting of concomitant medications in rheumatology randomized controlled trials of pharmacologic interventions
}

Michele Iudici, Carolina Riveros, Cem Gabay, Xavier Puéchal, Jean-Marie Berthelot, Philippe Ravaud

\section{To cite this version:}

Michele Iudici, Carolina Riveros, Cem Gabay, Xavier Puéchal, Jean-Marie Berthelot, et al.. Inadequate reporting of concomitant medications in rheumatology randomized controlled trials of pharmacologic interventions. Seminars in Arthritis and Rheumatism, 2019, 49, pp.453 - 458. 10.1016/j.semarthrit.2019.03.009 . hal-03488554

\section{HAL Id: hal-03488554 https://hal.science/hal-03488554}

Submitted on 21 Dec 2021

HAL is a multi-disciplinary open access archive for the deposit and dissemination of scientific research documents, whether they are published or not. The documents may come from teaching and research institutions in France or abroad, or from public or private research centers.
L'archive ouverte pluridisciplinaire HAL, est destinée au dépôt et à la diffusion de documents scientifiques de niveau recherche, publiés ou non, émanant des établissements d'enseignement et de recherche français ou étrangers, des laboratoires publics ou privés.

\section{(ㄷ)(1) $\$$}

Distributed under a Creative Commons Attribution - NonCommercial| 4.0 International 
1 Inadequate reporting of concomitant medications in rheumatology randomized 2 controlled trials of pharmacologic interventions.

3 Michele Iudici $^{1}$, Carolina Riveros ${ }^{1}$, Cem Gabay $^{2}$, Xavier Puéchal $^{3}$, Jean-Marie

4 Berthelot ${ }^{4}$, Philippe Ravaud ${ }^{1,5,6,7}$

5

${ }^{1}$ Methods of Therapeutic Evaluation Of Chronic Diseases (METHODS) team, INSERM, UMR 1153, Epidemiology and Biostatistics Sorbonne Paris Cité Research Center (CRESS), Paris, France.

2 Division of Rheumatology, Department of Internal Medicine Specialties, Geneva University Hospitals, Geneva, Switzerland.

${ }^{3}$ National Referral Center for Rare Systemic Autoimmune Diseases, Department of Internal Medicine, Hôpital Cochin, Assistance Publique-Hôpitaux de Paris (APHP), Université Paris Descartes, Paris, France.

${ }^{4}$ Service de rhumatologie, Hôtel-Dieu, CHU de Nantes, Nantes, France.

${ }^{5}$ Centre d'Epidémiologie Clinique, Hôpital Hôtel Dieu, Assistance Publique des Hôpitaux de Paris, Paris, France.

${ }^{6}$ Cochrane France, Paris, France.

${ }^{7}$ Department of Epidemiology, Columbia University, Mailman School of Public Health, New York, NY, United States.

\section{Corresponding author:}

Michele ludici, MD, PhD, Methods of Therapeutic Evaluation Of Chronic Diseases (METHODS) team, INSERM, UMR 1153, Epidemiology and Biostatistics Sorbonne Paris Cité Research Center (CRESS), Hôpital Hôtel-Dieu, 1 Place du Parvis Notre Dame, 75004 Paris, France. 
25 Current address: Division of Rheumatology, University Hospitals of Geneva, 26

26 Avenue de Beau-Séjour, 1211 Geneva 14, Switzerland

27 Email: michele.iudici@hcuge.ch

28 Word count: 2560 


\section{Abstract}

Objectives. To investigate how the use of pharmacologic concomitant medications (CMs) is planned and reported in rheumatology randomized controlled trials (RCTs).

Methods. We searched PubMed for RCTs on rheumatic diseases published in leading medical journals in the past 2 years. CMs potentially influencing primary outcome(s) were identified by a panel of experts and included in the analysis. Information on trial characteristics, how the use of CMs was planned and conducted throughout the trial were extracted. Data were summarized as number (\%) for qualitative, and median (range) for continuous variables.

Results. The 109 RCTs were mainly international (65\%), industry-funded (81\%) studies, including 267 CMs potentially influencing primary outcome(s). Forty-one RCTs (38\%) did not provide any data about the dosages of CMs allowed. Information on whether the intake of a permitted CM could have been modified during the study was missing in $24(22 \%)$ or incompletely reported in $23(21 \%)$ RCTs. As regards CMs use throughout the study, the baseline number of patients on CMs per arm and the mean baseline dosage was lacking in 18 (20\%) and 57 (52\%) trials, respectively. Ninety-five percent of RCTs did not provide any information on cumulative/mean exposure to CMs. Two of 109 trials described how many patients had modified CM dosage for reasons permitted by the protocol, and 3 reported the number of patients violating protocol because of an incorrect CM intake.

Conclusions. The use of CMs potentially impacting on primary outcome is poorly reported in rheumatology RCTs.

Keywords: randomized controlled trial, rheumatology, concomitant medications

\section{List of abbreviations}

RCTs. randomized controlled trials

NSAIDs. nonsteroidal anti-inflammatory drugs

CM. comedication

csDMARDs. conventional synthetic disease modifying anti-rheumatic drugs 


\section{Introduction}

Randomized controlled trials (RCTs) are the best tool to test the efficacy of pharmacologic treatments. Trial methodology and results should be accurately reported to ensure reproducibility, to correctly interpret study results and reduce wasteful research [1].

In addition to the intervention being studied, patients enrolled in RCTs often receive multiple treatments that could impact the outcome of interest because of pharmacokinetic interactions or because their known mechanism of action [2]. This is the case, for example of patients with rheumatic diseases enrolled in clinical trials taking concomitant oral glucocorticoids, nonsteroidal anti-inflammatory drugs (NSAIDs), analgesics or other medications.

Because these drugs tend to be modified after randomization according to the patient's status, the efficacy of the intervention under study, and the observed adverse events, their use should be carefully documented because between-arms differences can distort study results [3].

Efforts have been made during the last years to provide clinical researchers tools to improve the quality of (co)intervention reporting in RCTs [4-6]. International guidelines have underlined the importance to take into account the impact of comedications (CMs) in clinical trials [7]. Additionally, following the CONSORT 2010 and the SPIRIT 2013 statements, an extension tool entitled TIDieR (Template for Intervention Description and Replication)[5] has been more recently developed with the specific objective to further improve the completeness of reporting and ultimately the replicability of interventions in sufficient detail. The TIDieR statement emphasizes the need to carefully describe all interventions, including concomitant medications. Moreover, guidance to improve the description of some of the main concomitant drugs used in rheumatology has been provided in past years for some medications $[8,9]$.

Nevertheless, despite the availability of these tools, it seems that the description of concomitant treatment is still poor [10-12], and that this topic is not given much attention in medical literature. Issues of feasibility and the complexity of the analysis 
to be performed to adjust for covariate imbalance arising post-randomization could be potential explanations.

We performed this review to understand how the use of pharmacologic co-treatments is planned and reported in rheumatology RCTs published in leading journals. We decided to focus our attention only on concomitant treatments whose use was permitted by the trial and supposed to influence outcome.

\section{Materials and methods}

As the study did not concern human or clinical data, we did not record the protocol on PROSPERO. We followed the reporting guidelines for meta-analyses and systematic reviews of randomized controlled trials, Preferred Reporting Items for Systematic Reviews and Meta-Analyses (PRISMA) statement [13].

\subsection{Search strategy}

We performed an electronic search of MEDLINE via PubMed on 03 February 2018 to identify RCTs published in the five highest impact factor rheumatology [Annals of the Rheumatic Diseases, Arthritis \& Rheumatology, Rheumatology (Oxford), Seminars in Arthritis and Rheumatism, Osteoarthritis and Cartilage] and general and internal medicine [NEJM, Lancet, JAMA, BMJ, Annals of Internal Medicine] journals in the last 2 years. We used the Cochrane Highly Sensitive Search Strategy for identifying randomized trials [14], combined to terms referring to rheumatic diseases to identify papers in internal medicine journals. The full search strategy and the list of rheumatic diseases selected within the general and internal medicine journals can be found in online Supplementary File. Journal Citation Reports® (Clarivate Analytics) was used to identify the five best journals for each category.

\subsection{Eligibility criteria}

Types of studies. RCTs investigating pharmacologic treatments were included. We defined a RCT as a clinical study randomly allocating participants to different interventions. Pharmacologic treatments were drugs or nutritional supplements. Reports of secondary publications of RCTs, nonrandomized studies, observational studies, and reports of studies pooling data from more than one RCT were excluded. 
128 Meeting abstracts were also excluded. Types of participants. Participants of any age 129 having a rheumatic disease. Types of interventions. Pharmacologic treatments. 130 Types of outcomes. All outcomes were included.

\subsection{Data collection}

All retrieved references were downloaded in Covidence. Two researchers (MI, CR) independently checked each title and abstract to exclude irrelevant papers. If the information was unclear or insufficient in the abstract, the full text article was retrieved to confirm eligibility. The same reviewers then independently examined fulltext articles to determine eligibility. Consensus was reached by discussion in case of disagreement. A third reviewer was available in case of unsolved disagreement. We documented the primary reason for exclusion of full-text articles.

2.4 Identification of concomitant treatments and definition of comedication potentially influencing the primary outcome.

Information on CMs (i.e. each drug whose use was allowed in the trial apart from those under investigation) was obtained by reading full-text and supplementary material provided by authors. Three expert rheumatologists in the field (JMB, CG, XP) were chosen to independently assess the potential impact that each concomitant treatment could have had on primary outcome(s). They were provided the drug name (i.e. hydroxychloroquine) or category (i.e. TNF-alpha inhibitors), as found in full-text or supplementary data. They were blinded to all trial information except for the disease, the primary outcome(s), and time to primary outcome evaluation. For each concomitant medication they had to answer the following question: 'Can the intake of this CM during study influence at least one of the primary outcome(s)? (responses: definitely yes, maybe, definitely no). Only drugs for which an agreement on 'definitely yes' was reached by at least 2 experts, were defined 'concomitant medication potentially influencing the primary outcome' and further analyzed. 
RCTs with at least one reported CM potentially influencing the primary outcome were included. One author (MI) extracted the data from using a standardized form, and a second author (CR) checked the extracted data. Disagreements were discussed to reach consensus. From each study, the following characteristics were obtained: country of corresponding author, journal, year of publication, funding source (industry- or non-industry funded), disease, phase of development (phase $0, \mathrm{I}, \mathrm{II}, \mathrm{III}$, IV), type of comparator (placebo, active intervention, usual care, or no intervention), study design (parallel arm, cross-over, etc), primary outcome. A study was considered being industry-funded if the sponsor or one of the collaborators was industry.

For each $\mathrm{CM}$ included in the analysis we recorded the name and/or the drug category and whether authors provided information on: a) planned management of CM throughout the study (whether the possibility to use the drug was stated in eligibility criteria or in methods; whether the range of dose allowed, dose adjustment permitted and underlying reasons were reported; whether actions planned in case of protocol violations were acknowledged); b) observed use of the drug for each arm throughout the study (whether the number of patients taking CM at enrolment, at last visit and during the study was reported; whether the CM dosage at baseline, the cumulative/mean dosage were reported; whether the number of patients who modified drug dosage within the permitted range was described; whether reasons leading to dosage modification were reported; whether information on adherence to $\mathrm{CM}$ or on the occurrence of $\mathrm{CM}$-related protocol violations were provided).

Moreover, we identified a minimal core set of information which authors should have provided to allow to estimate CM exposure over time, if not reported. This was composed by: a) number of patients on CM at baseline; b) CM dosage at baseline; and c) cumulative/mean intake over the study.

\subsection{Data analysis}

We performed a descriptive analysis. Data were summarized as number (percentage) for qualitative variables and median (interquartile range, range) for continuous variables, as appropriate. We calculated the number (percentage) of RCT where information for each item related to planning (i.e. range of dose allowed, conditions permitting dose modification) or reporting (i.e. number of patients taking 
192 the drug at baseline, cumulative/mean intake over time) was completely (reported for 193 all CMs), partly (reported for $>0 \leq 50 \%$ of $\mathrm{CMs}$ ) or never (reported for no $\mathrm{CM}$ ) provided. The same information was provided at drug level. Moreover, we calculated the number (percentage) of RCTs where the minimal set of information (described above) needed to estimate drug exposure over time was reported for all CMs.

\section{Results}

One-hundred and nine RCTs with a total of 267 CMs defined by the experts as 'potentially influencing the primary outcome' were selected (see flow diagram in Figure 1). They were mostly international $(n=71 ; 65 \%)$, industry-funded $(n=88 ; 81 \%)$ studies conducted in rheumatoid arthritis $(n=50 ; 47 \%)$ patients. The characteristics of the studies included and the main concomitant treatments described are shown in Table 1.

\subsection{Planned management for concomitant medications}

205

206

207

208

The list of permitted drugs found in the Methods section or in eligibility criteria (in published report or supplementary material) did not include all CMs retrieved in the Result sections in 19 (17\%) studies. The permitted dosage of CMs was missing in 41 $(38 \%)$ or partly reported in $25(23 \%)$ RCTs. Information on whether the intake of a given CM could have been modified during the study was not present in $24(22 \%)$ or incomplete in $23(21 \%)$ studies. Reasons for drug dosage modification were not available for all CMs in $40 \%$ of RCTs (Table 2 ).

\subsection{Reporting on concomitant medication use during the trial}

The number of patients on CMs in each arm at baseline was the information more often given for all CMs (62 RCTs; 57\%), but mean baseline dosage was reported for all permitted CMs in only 17 (15\%) of trials. Ninety-five percent of RCTs did not provide any information on cumulative/mean exposure to concomitant medications (information fully reported for all the arms in 6 trials). The mean highest initial dosage $(n=1 ; 1 \%)$, the number of patients still taking the drug at last observation $(n=5 ; 4 \%)$ and/or the respective dosage $(n=3 ; 3 \%)$ were rarely reported (Table 2). Two of 109 trials $(2 \%)$ described how many patients had modified CM dosage for reasons 
221 permitted by the protocol, and $3(3 \%)$ reported the number of patients violating

222 protocol because of an incorrect CM intake (Table 2).

223 The minimal core set of information (number of patients taking CM at baseline, mean 224 CM dosage and mean/cumulative exposure over time) allowing to estimate CM use per arm, was available for all CMs in 4 (4\%) studies. In 15 (14\%) RCTs, the baseline number of patients on $\mathrm{CM}$ for each arm together with the mean drug dosage were shown, but not data on drug exposure throughout the study was available.

\subsection{Reporting for the different classes of drugs}

Table 3 shows the information about the planned and reported use of each main class of drugs in overall sample. The range of dosages permitted was lacking in $95 \%$ of cases for NSAIDs, $85 \%$ for analgesics and $40 \%$ for oral glucocorticoids. As far as the use of CMs during the study, authors were less likely to report the number of patients at study entry on NSAIDs or analgesics than that of patients on oral glucocorticoids or on conventional synthetic disease modifying anti-rheumatic drugs (csDMARDs) (Table 3). The same applied for the mean/median baseline dosage (information reported for about $30 \%$ of oral glucocorticoids and csDMARDs and $<5 \%$ for analgesics and NSAIDs). The description of mean/cumulative dose over the trial was rarely reported for all the classes of CMs. Table S1 in Supplementary file provides detailed information about how the use of each class of concomitant dugs was planned and reported in rheumatoid arthritis, the disease with the highest number of published trials within the last 2 years in rheumatology.

\section{Discussion}

We found that the use of CMs able to potentially influence primary outcome(s) was poorly reported in rheumatology trials. In most of cases, the main study report or the supplementary material did not completely describe how CM had been (planned to be) used to ensure treatment reproducibility or to assess between-arms difference of treatment exposure. Range of dosages allowed, possibility to modify CM intake, or drug exposure over the study are only some of the pivotal aspects partially detailed or lacking in most RCTs. 
Close monitoring and adequate reporting of pharmacologic treatment is mandatory to precisely estimate the benefit/risk ratio of an intervention. The cointervention bias [2], occurring when CM use is imbalanced between the arms may hamper to show the benefit of an experimental treatment, or conversely erroneously suggest its superiority. Overlooking this point by not adequately reporting which CM are used and how, and therefore potentially failing to analyze between-arms differences could represent therefore a threat to study validity.

Based on the results of the present study, we believe that this issue would deserve more attention in rheumatology where the management of inflammatory or systemic autoimmune diseases very often requires the use of treatment strategies based on a combination of drugs. It is the case, for example of NSAIDs use in spondyloarthritis [8] or of glucocorticoids in systemic lupus erythematosus patients [9]. In rheumatoid arthritis, it is well established that concomitant methotrexate (MTX) improves efficacy of TNFi [15]. Many other examples could have been outlined.

Reports providing guidance on how to collect and reporting information on CMs in rheumatology have been published in last years and should represent a starting point to improve reporting on CMs. The Assessment of SpondyloArthritis International Society (ASAS) guidelines for reporting NSAIDs intake in clinical trials in axial spondyloarthritis recommended to report the type of NSAIDs, the dose and the percentage of days with intake over a certain period of time [9]. In 2009, a panel of experts from the European League against the Rheumatism (EULAR) aiming to identify the main points to consider for conducting clinical trials in systemic lupus erythematosus, identified the careful description of glucocorticoids and other immunosuppressors as a priority to preserve the study conclusion integrity [8].

More than one reason can explain why the reporting of $\mathrm{CMs}$ is not exhaustive in the sample of RCTs analyzed. First, issues of feasibility could play an important role. The collection of a large amount of data and the extra work for both patients and physicians have been recognized as a major barrier to patient's recruitment and to study completion $[16,17]$. Thus, it can be hypothesized that to minimize the risk of poor recruitment or high patient drop-out, the choice not to collect these data has been made at protocol stage. Second, while there is broad consensus on analytic techniques to use for adjusting covariates at baseline, the adjustment for post- 
randomization imbalance in covariates is more complex and can introduce additional source of error [18]. However, such adjustment may be warranted when covariates are strongly correlated with the outcome or when there is a high degree of treatment imbalance between arms [18-20].

This study has some limitations. First, we included RCTs published in a very short period and in selected journals. An analysis extended to a longer observation time or a wide range of journals could have possibly provided additional information. Second, we retrieved data from published papers and supplementary material. The analysis of study protocols and e-CRF could have provided more information on how CM use was planned and used. Therefore, study conclusions about how CMs utilization was planned should be interpreted with caution. Third, the choice to label the drugs as 'potentially influencing the primary outcome' on the basis of an expert's opinion can be questioned. However, given the results of the present study, we assume that performing the same survey on a different number of drugs would not have changed the main study conclusions.

In conclusion, the description of the use of concomitant medications in rheumatology RCTs is poor and can represent a threat to study conclusion validity. Efforts should be made to enhance trials reporting in the field.

Competing Interests. Dr. ludici, Dr. Riveros, Pr. Berthelot, Pr. Ravaud declare no conflict of interest. Pr. Gabay has received research grants and consultant fees from Roche, AbbVie, Pfizer, BMS, MSD, UCB, Sanofi, Celgene, Regeneron, Actelion, Novartis, AB2 Bio, Debiopharm. Dr. Puéchal has received speaking fees and/or honoraria from Pfizer, LFB and Roche. Dr. Puéchal has been investigator of studies in which rituximab was given free of charge. Dr Puéchal has declared congress inscription/travel/accommodations from Sanofi. No other relationships/conditions/circumstances that present a potential conflict of interest.

Funding. None. 
Authors contributions. MI, PR contributed to the conception and design of the work; MI, CR contributed to the acquisition of the data; MI, CR, CG, XP, JMB, PR contributed to the analysis and interpretation of data; MI, CR, CG, XP, JMB, PR contributed to draft the work and critically revise it for important intellectual content. All the authors gave final approval of the version submitted.

\section{References}

1. Glasziou P, Altman DG, Bossuyt P, Boutron I, Clarke M, Julious S, et al. Reducing waste from incomplete or unusable reports of biomedical research. Lancet. 2014;383:267-76.

2. Sackett DL. Clinician-trialist rounds: 5 . Cointervention bias--how to diagnose it in their trial and prevent it in yours. Clin Trials. 2011;8:440-2.

3. Pagnoux C, Dechartres A, Giraudeau B, Seror R, Guillevin L, Ravaud P. Reporting of corticosteroid use in systemic disease trials: evidence from a systematic review of the potential impact on treatment effect. Arthritis Care Res. 2010;62:10028.

4. Begg C, Cho M, Eastwood S, Horton R, Moher D, Olkin I, et al. Improving the quality of reporting of randomized controlled trials. The CONSORT statement. Jama. 1996;276:637-9.

5. Hoffmann TC, Glasziou PP, Boutron I, Milne R, Perera R, Moher D, et al. Better reporting of interventions: template for intervention description and replication (TIDieR) checklist and guide. Bmj. 2014;7(348).

6. Schulz KF, Altman DG, Moher D. CONSORT 2010 statement: updated guidelines for reporting parallel group randomised trials. Bmj. 2010;23(340).

7. ICH Harmonised Tripartite Guideline: Structure and content of clinical study reports

E3.

Step

4.

http://www.ich.org/fileadmin/Public_Web_Site/ICH_Products/Guidelines/Efficacy/E3/ E3_Guideline.pdf.1995.

8. Gordon C, Bertsias G, loannidis JP, Boletis J, Bombardieri S, Cervera R, et al. EULAR points to consider for conducting clinical trials in systemic lupus erythematosus. Ann Rheum Dis. 2009;68:470-6. 
342 9. Dougados M, Simon P, Braun J, Burgos-Vargas R, Maksymowych WP, Sieper $J$, et al. ASAS recommendations for collecting, analysing and reporting NSAID intake in clinical trials/epidemiological studies in axial spondyloarthritis. Ann Rheum Dis. 2011;70:249-51.

10. Mahfoud F, Bohm M, Baumhakel M. Inadequate reporting of concomitant drug treatment in cardiovascular interventional head-to-head trials. Clin Cardiol. 2012;35:255-6.

11. Duff JM, Leather H, Walden EO, LaPlant KD, George TJ, Jr. Adequacy of published oncology randomized controlled trials to provide therapeutic details needed for clinical application. J Natl Cancer Inst. 2010;102:702-5.

12. Glasziou P, Meats E, Heneghan C, Shepperd S. What is missing from descriptions of treatment in trials and reviews? Bmj. 2008;336(7659):1472-4. 13. Moher D, Liberati A, Tetzlaff J, Altman DG. Preferred reporting items for systematic reviews and meta-analyses: the PRISMA statement. Ann Intern Med. 2009;151:264-9.

14. Glanville JM, Lefebvre C, Miles JN, Camosso-Stefinovic J. How to identify randomized controlled trials in MEDLINE: ten years on. $J$ Med Libr Assoc. 2006;94:130-6.

15. Klareskog L, van der Heijde D, de Jager JP, Gough A, Kalden J, Malaise M, et al. Therapeutic effect of the combination of etanercept and methotrexate compared with each treatment alone in patients with rheumatoid arthritis: double-blind randomised controlled trial. Lancet. 2004;363:675-81.

16. Mahmud A, Zalay O, Springer A, Arts K, Eisenhauer E. Barriers to participation in clinical trials: a physician survey. Curr Oncol. 2018;25:119-125.

17. Rendell JM, Merritt RD, Geddes JR. Incentives and disincentives to participation by clinicians in randomised controlled trials. Cochrane Database Syst Rev. 2007;(2):MR000021.

18. Heller G. An adjustment for a post-randomization variable in the comparison of two treatments for survival. Stat Med 2001;20:3475-85. 2005;15:593-603. 
373 20. Berger VW. A review of methods for ensuring the comparability of comparison 374 groups in randomized clinical trials. Rev Recent Clin Trials 2006;1:81-6.

375

376

377

378

379

380

381

382

383

384

385

386

387

388

389

390

391

392

393

394

395

396

397

398

399

400 
401 Tables

402 Table 1. Main features of the 109 studies included.

\begin{tabular}{ll}
\hline Study feature & n (\%)
\end{tabular}

\section{Journal}

Ann Rheum Dis

$48(44)$

Arthritis Rheumatol

$34(31)$

Lancet

$9(8)$

NEJM

$8(7)$

Osteoarthritis Cartilage

$4(4)$

Rheumatology

JAMA

$1(1)$

Ann Intern Med

$1(1)$

Seminars Arthritis Rheumatism

Industry funded

$88(81)$

International

Phase

Phase I, I/II, II

Phase II/III, III, IV

Not reported

\section{Study design}

Parallel group

$108(99)$

Crossover

Primary outcome

Binary

Continuous 36 (28)

Time to event $10(8)$

\section{Disease}

Rheumatoid arthritis

Connective tissue diseases

$18(17)$

Spondyloarthritis

$11(10)$

Gout/hyperuricemia

$8(7)$

Vasculitides

$8(7)$

Osteoarthritis

7 (6) 
Other

7 (6)

\section{Concomitant medications ${ }^{*}$}

csDMARDs

$119(44)$

Oral glucocorticoids

$80(30)$

Analgesics

$26(10)$

NSAIDs

$18(7)$

Others

$24(9)$

403

404

* number of concomitant medications across the studies; csDMARDs, conventional synthetic

405 disease-modifying anti-rheumatic drugs; NSAIDs, nonsteroidal anti-inflammatory drugs.

406

407

408

409

410

411

412

413

414

415

416

417

418

419

420

421

422

423

424 
425 Table 2. Information about the planned management and the use of concomitant 426 medications in rheumatology RCTs.

\begin{tabular}{|c|c|c|c|}
\hline & & $\begin{array}{l}\text { RCTs } \\
\mathrm{N}=109 \\
\end{array}$ & \\
\hline Information about CMs in RCTs & $\begin{array}{l}\text { reported for all } \\
\text { CMs }\end{array}$ & $\begin{array}{c}\text { reported for }>0 \leq 50 \% \\
\text { of } \mathrm{CMs}\end{array}$ & $\begin{array}{l}\text { reported for no } \\
\text { CMs }\end{array}$ \\
\hline \multicolumn{4}{|l|}{ Planned use } \\
\hline $\begin{array}{l}\text { The possibility to use the drug is clearly stated in } \\
\text { eligibility criteria or in method section }\end{array}$ & $90(82)$ & $6(5)$ & $7(6)$ \\
\hline Range of dose allowed & $28(26)$ & $25(23)$ & $41(38)$ \\
\hline $\begin{array}{l}\text { Information on dose modification allowed during } \\
\text { study period }{ }^{\wedge}\end{array}$ & $62(57)$ & $15(14)$ & $24(22)$ \\
\hline $\begin{array}{l}\text { Information on conditions permitting dose } \\
\text { modifications }{ }^{*}\end{array}$ & $31 / 52(60)$ & $1 / 52(2)$ & $11 / 52(21)$ \\
\hline \multicolumn{4}{|l|}{ Use during the trial } \\
\hline $\mathrm{N}$ of patients taking the $\mathrm{CM}$ at baseline in each arm & $62(57)$ & $14(13)$ & $20(18)$ \\
\hline Mean or median dosage at baseline for each arm & $17(15)$ & $26(24)$ & $57(52)$ \\
\hline Highest dosage at baseline for each arm & $1(1)$ & $0(0)$ & $108(99)$ \\
\hline $\begin{array}{l}\text { Cumulative/mean intake over the study period for } \\
\qquad \text { each arm }\end{array}$ & $6(5)$ & $4(4)$ & $99(91)$ \\
\hline $\mathrm{N}$ of patients taking the $\mathrm{CM}$ at last visit in each arm & $5(4)$ & $2(2)$ & $94(86)$ \\
\hline $\begin{array}{l}\text { Mean or median CM dosage at last visit in each } \\
\qquad \text { arm }\end{array}$ & $3(3)$ & $4(4)$ & $102(93)$ \\
\hline $\begin{array}{l}\mathrm{N} \text { of patients who modified the dosage for reasons } \\
\text { permitted by the trial }\end{array}$ & $2(2)$ & $0(0)$ & $107(98)$ \\
\hline Reasons leading to modify dosage & $0(0)$ & $0(0)$ & $109(100)$ \\
\hline $\begin{array}{l}\mathrm{N} \text { of patients who violated the protocol because of } \\
\text { incorrect } \mathrm{CM} \text { intake }\end{array}$ & $3(3)$ & $0(0)$ & $106(97)$ \\
\hline Reasons for protocol violation & $1(1)$ & $0(0)$ & $108(99)$ \\
\hline
\end{tabular}

428 If not otherwise specified, results refer to the number (\%) of randomized controlled trials 429 (RCTs); ^ studies reporting whether drug dosage was required to be kept stable are included 430 in this group, even if dosages ranges were not specified; *for trials where the dosage of at 431 least one concomitant medication was permitted to be modified through the study; RCTs, 432 Randomized controlled trial; $\mathbf{C M}$, Comedication. 


\section{$\mathrm{N}$ of $\mathrm{CMs}$}

\begin{tabular}{|c|c|c|c|c|c|}
\hline & $\begin{array}{c}\text { Total } \mathrm{N} \text { of } \\
\text { CMs in } \\
\text { RCTs } \\
\text { included } \\
\mathrm{N}=267 \\
\end{array}$ & $\begin{array}{l}\text { csDMARDs } \\
\qquad \mathrm{N}=119\end{array}$ & $\begin{array}{l}\text { Oral GC } \\
\mathrm{N}=80\end{array}$ & $\begin{array}{l}\text { Analgesics } \\
\qquad \mathrm{N}=26\end{array}$ & $\begin{array}{l}\text { NSAIDs } \\
\mathrm{N}=18\end{array}$ \\
\hline Planned use & & & & & \\
\hline $\begin{array}{l}\text { The possibility to use the drug is clearly } \\
\text { stated in eligibility criteria or in method } \\
\text { section }\end{array}$ & $241(90)$ & $112(94)$ & $69(86)$ & $24(89)$ & $16(89)$ \\
\hline Range of dose allowed & $112(42)$ & $55(46)$ & $49(61)$ & $4(15)$ & $1(5)$ \\
\hline $\begin{array}{l}\text { Information on dose modification } \\
\text { allowed during study period }\end{array}$ & $185(69)$ & $81(68)$ & $58(72)$ & $21(78)$ & $12(67)$ \\
\hline \multicolumn{6}{|l|}{ Use during the trial } \\
\hline $\begin{array}{l}\mathrm{N} \text { of patients taking the } \mathrm{CM} \text { at baseline } \\
\text { in each arm }\end{array}$ & $191(71)$ & $104(87)$ & $62(77)$ & $1(4)$ & $9(50)$ \\
\hline $\begin{array}{c}\text { Mean or median dosage at baseline for } \\
\text { each arm }\end{array}$ & $70(26)$ & $37(31)$ & $28(35)$ & $0(0)$ & $1(5)$ \\
\hline Highest dosage at baseline for each arm & $4(1)$ & $1(1)$ & $2(2)$ & $0(0)$ & $0(0)$ \\
\hline $\begin{array}{l}\text { Cumulative/mean dosage over the study } \\
\text { period for each arm }\end{array}$ & $10(4)$ & $0(0)$ & $9(11)$ & $0(0)$ & $1(5)$ \\
\hline $\begin{array}{l}\mathrm{N} \text { of patients taking the } \mathrm{CM} \text { at last visit } \\
\text { in each arm }\end{array}$ & $9(3)$ & $3(2)$ & $6(7)$ & $0(0)$ & $0(0)$ \\
\hline $\begin{array}{l}\text { Mean or median CM dosage at last visit } \\
\text { in each arm }\end{array}$ & $8(3)$ & $3(2)$ & $5(6)$ & $0(0)$ & $0(0)$ \\
\hline
\end{tabular}

437 If not otherwise specified, results refer to the number (\%) of cointerventions. ^ studies $^{\circ}$

438 reporting whether drug dosage was required to be kept stable are included in this group,

439 even if dosages ranges were not specified ; CM, comedication. GC, glucocorticoids;

440 NSAIDs, nonsteroidal anti-inflammatory drugs; csDMARDs, conventional synthetic disease-

441 modifying anti-rheumatic drugs. 
445 Figure 1. Flow-chart of study selection.

446

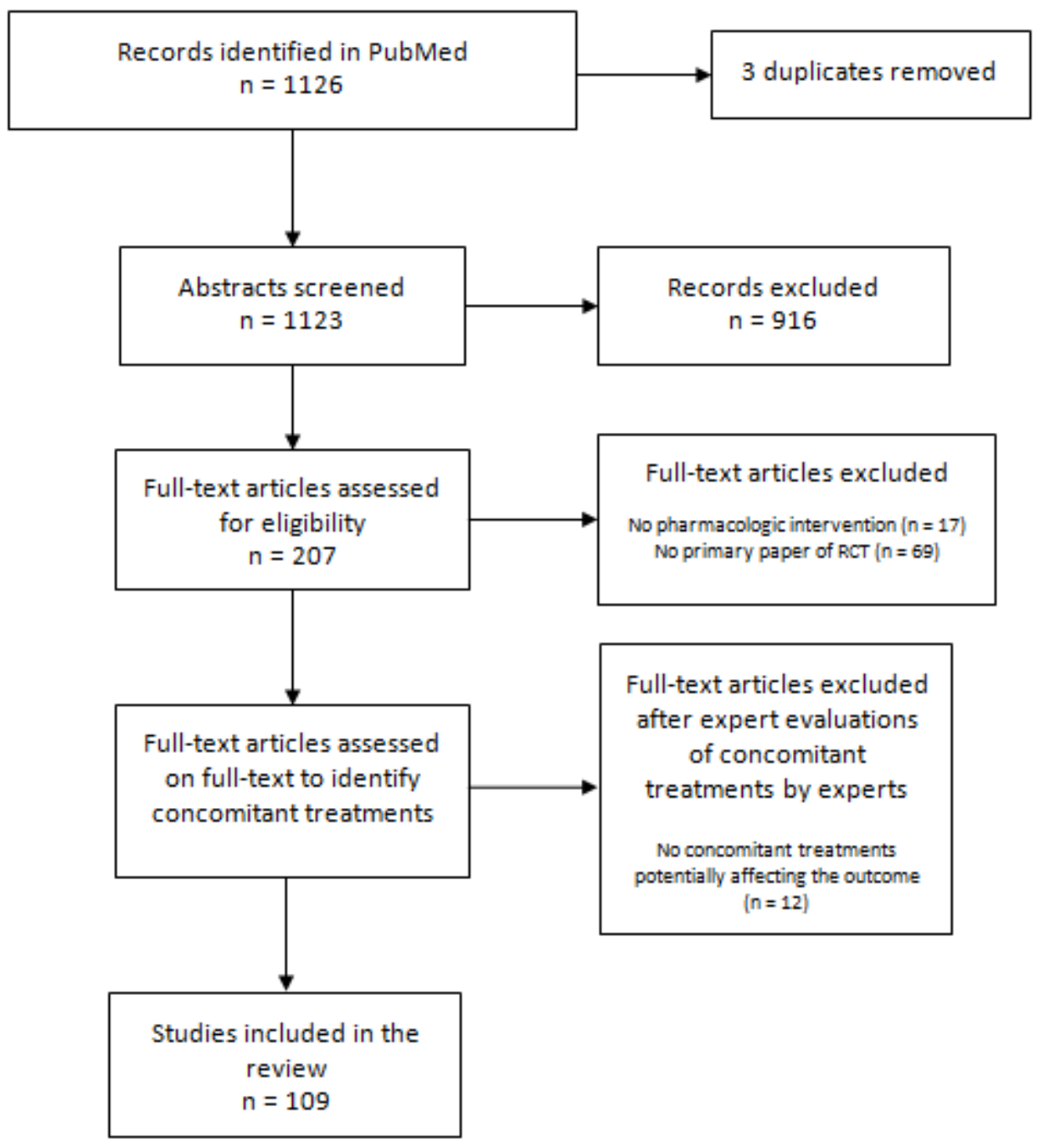

447 